\title{
The aspirations of Japanese undergraduate students: Prospective study on their effects on depression and anxiety and mediation by adult attachment
}

\author{
Toshinori Kitamura $^{1,2}$, Nobuhiko Kijima ${ }^{3}$, Mika Suzuki Takeuchi ${ }^{4}$, Atsuko Tomoda ${ }^{5}$ \\ ${ }^{1}$ Kitamura Institute of Mental Health Tokyo, Tokyo, Japan \\ ${ }^{2}$ Department of Psychiatry, Graduate School of Medicine, Nagoya University, Nagoya, Japan \\ ${ }^{3}$ Psychological Laboratory, Keio University, Yokohama, Japan \\ ${ }^{4}$ Faculty of Humanities and Social Sciences, Jissen Women's University, Tokyo, Japan \\ ${ }^{5}$ Department of Psychology, Department of Psychology, Faculty of Human and Social Studies, Saitama Institute of Technology, Fukaya, \\ Japan
}

\section{Email address: \\ kitamura@institute-of-mental-health.jp (K. Toshinori)}

\section{To cite this article:}

Toshinori Kitamura, Nobuhiko Kijima, Mika Suzuki Takeuchi, Atsuko Tomoda. The Aspirations of Japanese Undergraduate Students: Prospective Study on their Effects on Depression and Anxiety and Mediation by Adult Attachment. Psychology and Behavioral Sciences. Vol. 3, No. 5, 2014, pp. 171-178. doi: 10.11648/j.pbs.20140305.14

\begin{abstract}
The present study examined the connections among aspiration, depression, anxiety, attachment and stressful life events in a sample of 405 Japanese undergraduate students. At Time1 (T1), participants' Dysphoric Mood (depression and anxiety rated by the Hospital Anxiety and Depression Scale: HADS), Adult Attachment (rated by the Relationship Questionnaire) and factor-analysis-derived two dimensions of Aspiration (rated by the Aspiration Index) - Agency and Communality Aspirations - were measured. Three months later (Time2 [T2]), participants completed the HADS again and reported the impact of their most negative life event occurring during the interval between T1 and T2. After controlling for the direct path from T1 to T2 Dysphoric Mood, both T1 Dysphoric Mood and low Communality Aspiration predicted insecure Adult Attachment, which predicted the perception of Agency Stress, which in turn predicted T2 Dysphoric Mood. These findings suggest that adolescents' Communality Aspiration is important in maintaining secure attachment and indirectly subsequent healthy psychological adjustment.
\end{abstract}

Keywords: Aspiration, Depression, Anxiety, Stressful Life Events, Adult Attachment

\section{Introduction}

Well-being and life satisfaction depend on the balance between aspiration and attainment (Plagnol\&Easterlin 2008). Human behaviours are goal-directed. Aspiration is the desire for personal achievement. It leads to motivation and determination that are necessary in shaping the direction of one's life. This is particularly important during adolescence (Massey et al. 2008). Successful goal attainment is related to health and well-being (Massey et al. 2008), whereas different types of aspirations are related to psychological adjustment and maladjustment. For example, Kasser and Ryan (1993) reported that aspiration for financial success was associated negatively with well-being and positively with depression and anxiety in cross-sectional studies. Further, Kasser and Ryan (1996) noted that depression was associated with low intrinsic aspiration (i.e., affiliation, community feeling, physical fitness, and self-acceptance) and high extrinsic aspiration (i.e., financial success, social recognition, and appealing appearance). These investigations were, however, cross-sectional and therefore causal direction remains to be determined in prospective studies.

Because ambitions differ among individuals, threat to or loss of specific goals may result in differing levels of stress across any given population. The extent to which events are perceived as threatening or stressful may depend on the degree to which these events are related to an individual's aspirations. Similarly, the degree of the stress induced by a life event may be determined by the magnitude of an individual's aspirations in this area. For example, a row with a friend may be perceived as very stressful to people who place much value on affiliation or community feeling, but 
less so to those who place little value on these aspiration domains. Hence, perceived stress may be determined by varying impacts of different aspiration domains. Further, the psychological reaction of an individual to a stressful event may be stronger if the aspiration domain associated with that event is a domain the individual values highly as a goal.

In addition to aspiration, adult attachment has been viewed as important to the mental health of adolescents. Insecure adult attachment in university students predisposed them to developing depression in a longitudinal study (Liu et al. 2009). Secure attachment with an intimate partner is an important component of the affiliation aspiration in adolescents and young adults. Adult attachment in young people may be partly determined by their affiliation aspiration. It may also be that secure attachment reduces the stress of life events. Thus, the reported link between specific types of aspiration and psychological maladjustment such as depression and anxiety may be mediated by insecure adult attachment.

Life satisfaction, aspiration, and adult attachment may be culture-specific. For example, Kim et al. (2003) studied Korean and American university students and found that self-actualization, vitality, and happiness were higher among the American students than the Korean students. These measurements were positively correlated with relative independence (as against interdependence) among students in both countries. It has been reported that life values are culture-specific (Oishi et al. 1999; Oishi et al. 1998). There has been little study among Japanese populations regarding the links between life satisfaction, aspiration, and adult attachment. The current report is a prospective study of the effects of Japanese university and college students' aspiration styles on dysphoric mood (depression and anxiety) via their adult attachment. Our research questions are:

1. How many aspiration domains exist among Japanese young people?

2. Are aspirations associated with current dysphoric mood (depression and anxiety)?

3. Is adult attachment predicted by aspiration styles and current dysphoric mood?

4. Is the perception of stressful life events predicted by aspiration styles and dysphoric mood?

5. Is the effect described in (4) mediated by adult attachment?

6. Is dysphoric mood at the follow-up time point predicted by the perception of stressful life events?

7. Is dysphoric mood at the follow-up time point predicted directly by baseline dysphoric mood, aspiration, and adult attachment?

8. Are the above variables influenced by age and gender?

\section{Method}

\subsection{Participants}

Students of two universities and one college in Tokyo metropolitan area were solicited to participate in this two-wave prospective study on mental health. This study was based on the "catch as catch can" principle. A total of 633 students participated in the first wave (Time 1 [T1]). Of these, $457(72 \%)$ also took part in the second wave (Time 2 [T2]) three months later. We excluded students aged 25 or higher because a main purpose of this study was to examine the aspirations of adolescents and young adults. This resulted in 402 students presenting a full set of data available for analyses. There were 199 men and 203 women witha mean (SD) age of $19.1(0.8)$ years. Men $($ mean $=19.4, \mathrm{SD}=0.9)$ were slightly but significantly $(P<.001)$ older than women $($ mean $=18.9, \mathrm{SD}=0.6)$.

\subsection{Measures}

\subsubsection{Life Aspirations}

We used the Aspiration Index (AI: Kasser\&Ryan 1993). This is a self-report of aspiration tendencies. This includes 32 possible aspirations each rated with two 5 -point scales $(0-$ not at all to $4-$ very high). The aspiration domains include seven areas containing four to five items each: Self-Acceptance (SA; a sample item is "You will know and accept who you really are"), Affiliation (AF;a sample item is "You will share your life with someone you love"), Community Feeling (CF;a sample item is "You will work for betterment of society"), Physical Fitness (PF; a sample item is "You will be physically healthy"), Social Recognition (SR; a sample item is "You will be admired by many people"), Appealing Appearance (AA; a sample item is "You will successfully hide the signs of aging"), and Financial Success (FS; a sample item is "You will have a job with high social status"). Each item is rated on two aspects: (a) personal importance ("importance") and (b) the chance of attaining the item in the future ("chance"). The AI was translated by one of the present authors(T.K.) after obtaining permission from the original author. It was retranslated back into English by someone unaware of the original English wording in order to verify the Japanese phrases. In this study, two items were selected for each dimension of aspiration in order to reduce the total number of items to 14 because of the page availability of the questionnaire which contained many other items. Thus, the possible score range of each dimension was 0 to 8 . In the present study, we analyzed only the scores of the personal importance aspects.

\subsubsection{Dysphoric Mood}

Emotional distress is assessed by the Hospital Anxiety and Depression Scale (HADS;Zigmond\&Snaith 1983). This is a 14-item self-report of cognitive symptoms of depression and anxiety. No items referring to somatic symptoms were originally incorporated. There are seven depression and seven anxiety items. The scale is rated from 0 (low depression or anxiety) to 3 (high depression or anxiety). We used the total score of the HADS, which could range between 0 and 42. A higher score indicates more severe dysphoric mood symptomatology. The reliability and validity of the HADS has been well established (Mykletun et al. 2001). The 
HADS was translated by one of the present authors(Kitamura 1993) after obtaining permission from the original author. It was retranslated into English by someone unaware of the original English wording to verify the Japanese phrases. The factor validity of the Japanese version of the HADS was reported by Matsudaira et al. (2009).

\subsubsection{Adult Attachment}

We used the Relationship Questionnaire (RQ; Bartholomew \& Horowitz 1991). This is a measure of adult styles of intimate attachment to another person. The RQ measures four categories of adult attachment (Secure, Fearful, Preoccupied, and Dismissing). It is composed of four paragraphs, each describing one attachment style. Participants were asked to rate the extent to which each description corresponded to their relationship with their partner with a 7-point scale ranging from "Does not apply to me at all" to "Applies to me very much". If they had no definite partner, they were requested to imagine a close opposite-sex person in answering the question. The questionnaire's reliability (Bartholomew \& Horowitz 1991) and validity (Griffin \& Bartholomew 1994) have been reported. With the permission of Dr. Bartholomew, the RQ was translated into Japanese (T.K.). As the Japanese version of the RQ was very short, it was not retranslated back into English to confirm whether the translation was consistent with the original meaning. In this study, we calculated the total score of the RQ by subtracting the scores of the three insecure attachment styles (Fearful, Preoccupied, and Dismissing) from the score of the Secure attachment style. A higher total RQ score suggests more secure adult attachment.

\subsubsection{Stressful Life Events}

A list of 36 stressful life events that university students are likely to encounter was presented and students were asked to report whether they had experienced any of them and, if answered affirmatively, were asked to rate the impact of the event using a scale between -100 (most undesirable) and +100 (most desirable). Then they were asked to choose the event with the worst impact and rate its impact in terms of loss of or threat to importance of each of the seven domains of aspiration with 5 -point scale $(0$ - not at all; 4 - very much so). Thus, there were 14 questions (two categories (loss and threat) for each of the seven domains (SA, AF, CF, PF, SR, AA, and FS)).

\subsection{Procedures}

A set of questionnaires was distributed and returned during a class on two occasions with an interval of about three months.

\subsection{Statistical Analyses}

First, using half of the sample population $(n=315)$ we performed an exploratory factor analysis (EFA) of the significance aspects of the seven AI dimension scores of the seven AI dimension scores of the significance aspect with Promax rotation. The number of factors was determined by the screen test (Cattell 1996) as well as Kaiser criterion. The robustness of the factor structure derived from this EFA was confirmed by a confirmatory factor analysis (CFA) using the second half of the study population $(n=306)$. The fit of each model with the data was examined in terms of chi-squared (CMIN), comparative fit index (CFI), and root mean square error of approximation (RMSEA). According to conventional criteria, a good fit would be indicated by $\mathrm{CMIN} / \mathrm{df}<2$, CFI $>$ 0.97, and RMSEA $<0.05$, and an acceptable fit by CMIN/df $<3$, CFI $>0.95$, and RMSEA $<0.08$ (Schermelleh-Engell 2003).

The next stage of our analysis was to determine the predictive validity of the AI in the Japanese university student population. The criterion variable was T2 Dysphoric Mood (anxiety \& depression). After calculating the means and SDs of all the variables used in this study, as well as the correlations between them, we set up a path model (Fig. 2).In this model, we hypothesized that (1) Both T1 and T2 Dysphoric Mood would be upper latent variables for HADS Depression and Anxiety; (2) the error variables of T1 Dysphoric Mood and Agency and Communality Aspirations would covary with each other; (3) Adult Attachment would be predicted by T1 Dysphoric Mood and Agency and Communality Aspirations; (4) the perception of stressful life events occurring during the follow-up period would be predicted by Adult Attachment as well as T1 Dysphoric Mood and Agency and Communality Aspirations; (5) T2 Dysphoric Mood would be predicted by the perception of stressful life events, Adult Attachment, T1 Dysphoric Mood and Agency and Communality Aspirations; and (6) Age and Gender would predict all the variables measured at T1 as well as the perception of stressful life events occurring during the follow-up period.

We used SPSS18 and AMOS 18.0.

\section{Results}

\subsection{Factor Structure of the AI}

Table 1. Means and SDs of the AI items and their factor structure in a randomly split half of the student population $(n=318)$.

\begin{tabular}{llll}
\hline AI items & Mean (SD) & Factor & \\
\hline & & 1 & 2 \\
Financial Success & $3.6(1.5)$ & .84 & -.11 \\
Physical Fitness & $4.9(1.6)$ & .79 & -.18 \\
Community Feeling & $4.1(1.9)$ & .67 & .08 \\
Appealing Appearance & $4.6(1.5)$ & .54 & .23 \\
Affiliation & $6.3(1.4)$ & -.10 & .79 \\
Self-Acceptance & $7.0(1.1)$ & -.07 & .77 \\
Social Recognition & $4.5(1.4)$ & .37 & .48 \\
\% of variance explained & --- & 36.5 & 17.3 \\
\hline
\end{tabular}

Factor loadings $>.45$ are in bold.

An EFA using a randomly split half of the student sample yielded a two-factor structure (Table 1). This met the requirement of both scree test and Kaiser criterion. The AI 
dimensions with a high loading $(>.45)$ on the first factor included Financial Success, Physical Fitness, Community Feeling, and Appealing Appearance. These AI dimensions represented being physically vigorous and looking attractive so that one could perform successful jobs and be positively appraised by surrounding people. Thus, we termed this factor "Agency Aspiration". The AI dimensions with a high loading on the second factor included Affiliation, Self-Acceptance, and Social Recognition. These AI dimensions represented being affectionate, aware of one's own responsibility, and respected by the society. Thus, we termed this factor
“Communality Aspiration".

In order to confirm the robustness of the factor structure, we performed a CFA using the second half of the student population (Fig. 1). Good fit was observed for the original model. We observed a moderate covariance between the latent factors of Agency Aspirations and Communality Aspirations. Thus, we created two composite variables Agency Aspirations and Communality Aspirations - for further analyses through summation of all the dimension scores belonging to each factor using z-transformation.

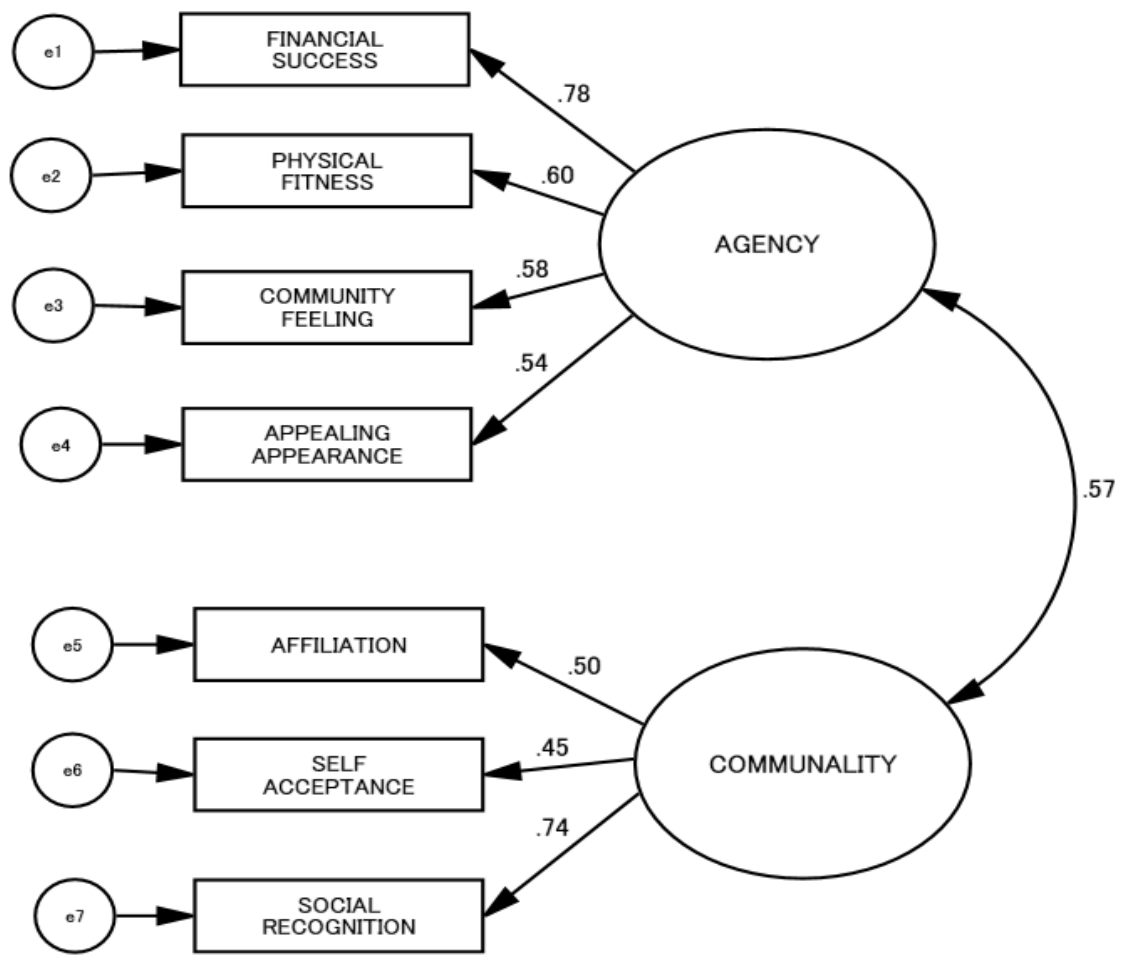

$$
\begin{aligned}
& \text { CHI-SQUARED }=34.934 \\
& D F=13 \\
& \text { CFI }=.942 \\
& \text { RMSEA }=.074 \\
& 90 \% \text { CI RMSEA LOWEST }=.045 \\
& 90 \% \text { CI RMSEA HIGHEST }=.105
\end{aligned}
$$

\begin{tabular}{|c|c|c|c|c|c|c|c|c|c|}
\hline & 1 & 2 & 3 & 4 & 5 & 6 & 7 & 10 & 11 \\
\hline 1 T2 Dysphoric Mood & --- & & & & & & & & \\
\hline 2 Gender $(1-$ men; 2 -women $)$ & -.01 & --- & & & & & & & \\
\hline 3 Age & .01 & $-.30 * * *$ & --- & & & & & & \\
\hline 4 T1 Dysphoric Mood & $.62 * * *$ & .02 & -.03 & --- & & & & & \\
\hline 5 Adult Attachment & $-.34 * * *$ & $.11 * *$ & -.05 & $-.38 * * *$ & --- & & & & \\
\hline 6 Agency Aspiration & $-.11 *$ & .02 & $.10 *$ & $-.16 * * *$ & $.17 * * *$ & --- & & & \\
\hline 7 Communality Aspiration & $-.13 *$ & $.14 * * *$ & -.05 & $-.17 * * *$ & $.24 * * *$ & $.35 * * *$ & --- & & \\
\hline 11 Communality Stress & $.13 * *$ & $.14 * * *$ & -.05 & $.10 *$ & -.05 & .03 & -.02 & $.51 * * *$ & --- \\
\hline $\mathrm{n}$ & 413 & 619 & 624 & 611 & 616 & 624 & 624 & 624 & 624 \\
\hline Mean (SD) & 11.1 & 1.5 & 19.2 & 11.5 & -4.1 & 0.0 & 0.0 & 3.6 & 3.5 \\
\hline SD & 5.9 & 0.5 & 0.8 & 5.8 & 4.4 & 1.0 & 1.0 & 5.6 & 5.4 \\
\hline
\end{tabular}

Figure 1. CFA of the AI items in another randomly split half of the subject population.

Table 2. Means and SDs of and intercorrelations between all the variables used in this study.

$* p<.05 ; * * p<.01 ; * * * p<.001$ 
Because the scores of dimensions of the AI showed two factors-Agency and Communality-, we then summed the scores assigned to Loss of and Threat to the importance of aspiration regarding the worst life event separately for the two aspiration dimensions-Agency and Communality. We called them Agency and Communality Stress scores. Agency and Communality Stress scores were all moderately correlated with each other (Table 2).

\subsection{Prediction of T2 Dysphoric Mood}

A correlation matrix of the variables used in this study showed that (1) T2 Dysphoric Mood was correlated significantly with T1 Dysphoric Mood, insecure Adult Attachment, lower Agency and Communality Aspirations, and Agency and Communality Stresses; (2) compared with men, women were younger, more secure in Adult Attachment, had higher Communality Aspiration, and were more impacted by Communality Stress; (3) older students scored higher in Agency Aspiration; (4) T1 Dysphoric Mood was correlated with insecure Adult Attachment and low Agency and Communality Aspirations; and (5) secure Adult Attachment was correlated with Agency and Communality Aspirations (Table 2).

Path analytic results of the hypothesized model are shown inFig. 2. The model resulted in good fit with the data: $\mathrm{CMIN} / \mathrm{df}=0.81, \mathrm{CFI}=1.000, \quad \mathrm{RMSEA}=.000 \quad(90 \% \mathrm{CI}$ 0.000-0.039). The error variable of T1 Dysphoric Mood was negatively correlated with those of both Agency and Communality Aspiration; the error variables of Agency and Communality Aspirations were intercorrelated; T1 Adult Attachment was predicted by concurrent Community Aspiration and low Dysphoric Mood; the error variables of Agency and Communality Stresses were predicted by T1 Dysphoric Mood and were intercorrelated whereas only Agency Stress predicted T2 Dysphoric Mood and it was predicted by insecure Adult Attachment; T1 Dysphoric Mood directly predicted T2 Dysphoric Mood. As expected, T1 Agency Aspiration was predicted by age while T1 Communality Aspiration and Communality Stress were predicted by female gender.

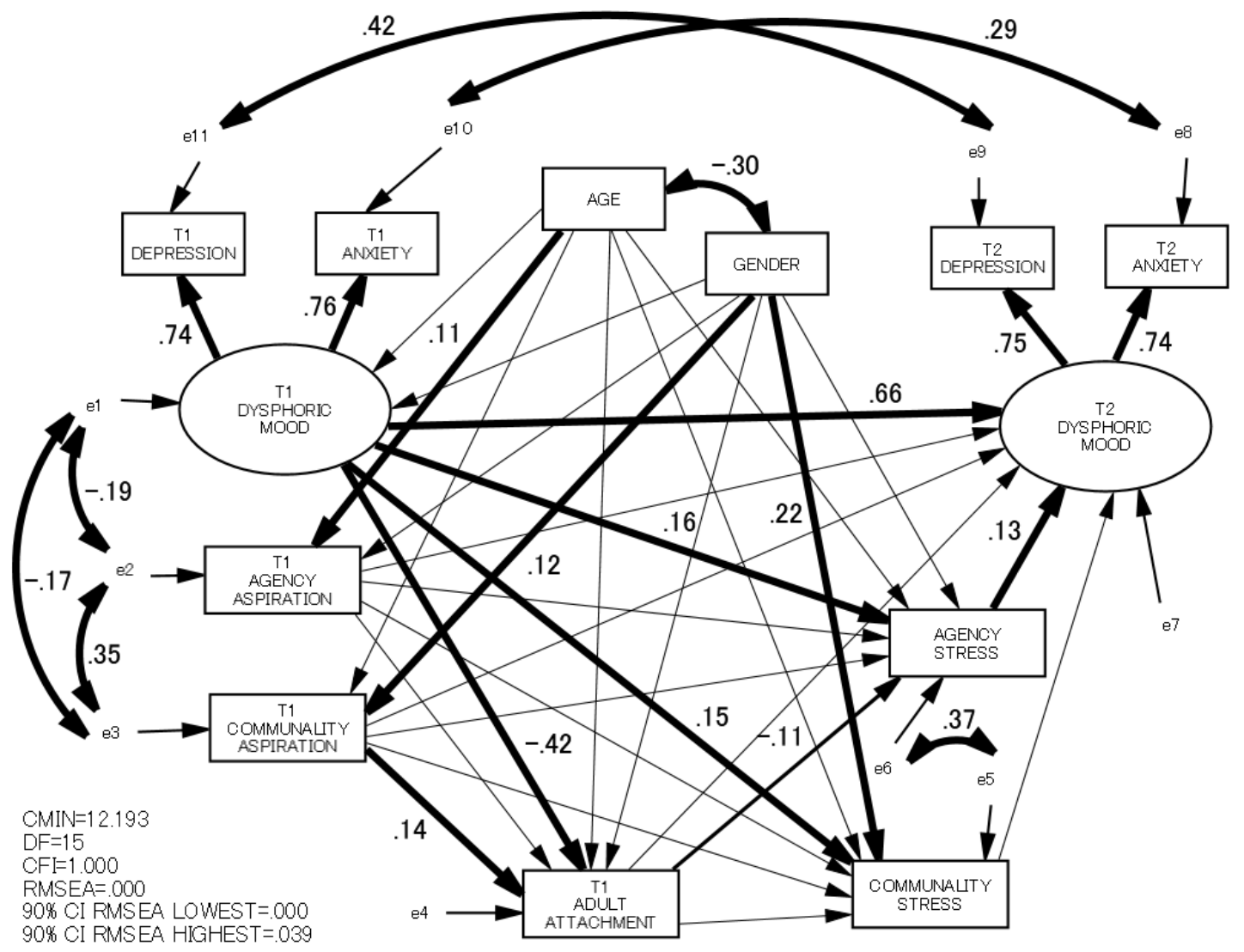

Figure 2. Path model Path estimates that are not significant are not described. Paths that significant $(P<.05)$ are in bold.

\section{Discussion}

The present study showed two aspiration domains among a Japanese university student population: one reflecting intrapersonal desires such as financial success and physical fitness, and the other reflecting interpersonal desires such as affiliation and self-acceptance. Kasser and Ryan (1996) proposed a theory-driven division of the seven aspiration dimensions into two domains-intrinsic and extrinsic. This division was confirmed in EFAs in both German and American college student populations (Schmuck et al. 2000). The results of our own EFA differed from these. While extrinsic dimensions including Financial Success and 
Appealing Appearance loaded high on the first factor, this factor was also loaded by Physical Fitness and Community Feeling, both intrinsic dimensions. On the other hand, intrinsic dimensions including Affiliation and Self-Acceptance loaded high on the second factor but this factor was loaded also by Social Recognition, an extrinsic dimension. Kim et al. (2003) used the AI in their study of Korean and American students. However, they did not examine whether the factor structures of the AI were the same between the two student populations. Studying more than 1,000 undergraduates in 15 countries (including East Asian countries China and South Korea, but not Japan), Grouzet et al. (2005) found that the 11dimensions of aspiration were consistently organized in a circumplex fashion. The circumplex was described by positing two axes: intrinsic (e.g., self-acceptance, affiliation) versus extrinsic (e.g., financial success, image) and self-transcendence (e.g., spirituality) versus physical (e.g., hedonism). The result of the present EFA does not fit well with the circumplex model. Our result may be culture specific. For example, motivation is under the influence of self-construal and the latter is culture specific (Markus \&Wurf 1987). While the self-construal of Americans is independent, that of Japanese is interdependent. In the Japanese culture, people's achievement is often evaluated by its meaning in the framework of the broader social network (Markus \&Kitayama 1991). Japanese may define values in terms of their concordance with what their society demands or with what they themselves desire. Thus, the domains of life values and goals may be classified by virtue of their direction: towards society or towards themselves.

Financial success, which was reported to be a predictor of psychological maladjustment in American studies (Kasser\& Ryan 1993, 1996), was not associated with depression or anxiety in this study. Rather, it was a part of a broader aspiration valuing one's ability, energy, and outward enthusiasm. The association between financial aspiration and psychological maladjustment in American undergraduates (Kasser\& Ryan 1993, 1996) may be explained by their low mindfulness. Mindfulness is open attention to and awareness of one's internal states and external events in the present. Brown et al. (2009) examined the gap between current and desired financial states (financial desire discrepancy) and found that the greater the gap the poorer the participant's subjective well-being. Moreover, the financial desire discrepancy was reduced by mindfulness. Given the definition of mindfulness, psychological maladjustment may result not from what people desire financially but how acceptable they perceive the gap between what they have and what they want. Accepting who and what one is a basis of East Asian philosophy. Hence, the degree of an East Asian's financial desire may be unrelated to his or her psychological maladjustment.

This study indicated that students high in either aspiration domain were more likely to be psychologically well. They were less likely to report subjective feelings of depression or anxiety. Students low in either aspiration domain may be more lethargic or less interested in important activities. However, the causal relationship between aspiration and well-being or dysphoric mood is impossible to determine based on cross-sectional data. One may lead to another or vice versa. Alternatively, the two may be parts of a single trait. Hence, determining how aspiration and current well-being predict future well-being is pivotal before the issue of causation can be resolved. Dysphoric mood during the follow-up phase correlated moderately with baseline dysphoric mood and mildly with the low aspiration level of the two types in our prospective study. This suggests that level of aspiration is, to some extent, causal in developing psychological well-being. However, the links between low Aspiration scores and follow-up Dysphoric Mood may be spurious due to rather robust association between the baseline and follow-up Dysphoric Mood scores. This study was unique in that we assessed psychological adjustment on two occasions and predicted the psychological adjustment at follow-up based on baseline Aspiration domains after controlling for baseline psychological adjustment. It was found that baseline aspirations indirectly predicted the follow-up psychological adjustment. The negative correlation between T1 Community Aspiration and T2 Dysphoric Mood disappeared in the path model possibly because confounded by T1 Dysphoric Mood.

This prospective study also demonstrated that secure adult attachment predicted later psychological well-being. This is in line with previous investigations (Armsden\& Greenberg 1987; Armsdenet al. 1990; Liu et al. 2009). Moreover, in this study the link between secure adult attachment and later psychological adjustment was mediated by lower degrees of Agency Stress. Agency Stress refers to either loss of or threat to what students value as belonging to themselves-financial values, physical fitness, and community feeling. It may be that either those with secure adult attachment are less likely to experience stressful life events related to agency values or that, although the rate of occurrence of such events does not vary, such people are likely to feel such events as less stressful. Becausesense of agency is associated with life satisfaction (Welzel\&Inglehart 2010), it may be loss of or threat to agencyfeeling that is stressful rather than those ofcommunalityfeeling. Communality values were not a factor On the other hand, secure attachment was predicted by high levels of Communality Aspiration. People valuing affiliation, self-acceptance, and social recognition are more likely to have secure adult attachment.

Our path model showed that the effects of aspiration on later psychological well-being was indirect and may be mediated by both more secure adult attachment and a lesser perception of stress related to agency values. In contrast, Liu et al. (2009) studied a Japanese university student population and found that insecure attachment and perceived life stress independently played a direct role in the onset of depression. This discrepancy may be due to differencesin length of the follow-up period. The interval between the baseline and follow-up assessments was one week in Liu et al.'s (2009) study while it was three months in this study. Various 
researchers have supported the "main effect" model in which secure attachment and life stress have a generalized separate impact on mental health (Kenny et al. 1998; Christensen et al. 2003). Other investigators have proposed the "buffering" model, in which secure attachment and perceived life stress attenuate each other's adverse effects, whereas in the absence of life stress, secure attachment has little impact on depression, and vice-versa (Hammen et al. 1995; Papini\&Roggman 1992; Petersen et al. 1994). Most past investigations used the impact of negative life events as a measure of the stress caused by these events. In the present study, we measured the impact of events in terms of loss of or threat to the life goals of the participants. The modes of effect of adult attachment, life events, as well as aspiration styles deserve further study.

Limitations of this study should be considered. We used depression and anxiety as measures of psychological adjustment. However, other psychological factors should also be studied before coming to a conclusion about the link between aspiration, adult attachment, stressors, and well-being. Further, it has been claimed that macrosocial factors such as the economic wealth of nations, the distribution of income, the extent of the welfare state and political freedom are relevant to life satisfaction (Haller \& Hadler 2006). We had no means of measuring such societal influences. All the information in this study was obtained from participating students and is therefore subject to shared informant bias. We used a short version of the AI. Use of the full version may have produced a different outcome. Another drawback of this study is a lack of measurement of aspiration at $\mathrm{T} 2$. It may be that aspiration change over the course of time. The relatively small number of participants should also be takeninto account. The age range was limited to young people and thus the findings may be specific to this life stage.

\section{Conclusions}

The present study suggests a flow of predictions from aspiration, adult attachment, perception of stressful life events, and final psychological adjustment. Adolescents' community aspiration is important in maintaining secure attachment and indirectly subsequent healthy psychological adjustment.

\section{References}

[1] Armsden, G. C., \& Greenberg, M. T. (1987). The Inventory of Parent and Peer Attachment: Individual differences and their relationships to psychological well-being in adolescence. Journal of Youth and Adolescence, 17, 427-454.

[2] Armsden, G. C., McCauley, E., Greenberg, M. T., Burke, P. M., \& Mitchell, J. R. (1990). Parent and peer attachment in early adolescent depression. Journal of Abnormal Child Psychology, 18(6), 683-697.

[3] Bartholomew, K., \&Horowiz, L. M. (1991). Attachment styles among young adults: A test of four-category model. Journal of
Personality and Social Psychology, 61, 226-244.

[4] Brown, K. W., Kasser, T., Ryan, R. M., Linley, P. A., \&Orzech, K. (2009). When what one has is enough: Mindfulness, financial desire discrepancy, and subjective well-being. Journal of Research in Personality, 43, 727-736.

[5] Cattell, R. B. (1996). The screen test for the number of factors. Multivariate Behavioral Research, 1, 245-276.

[6] Christensen, E. M., Gjerris, A., Larsen, J. K., Bendtsen, B. B., Larsen, B. H., Rolff, H., et al. (2003). Life events and onset of a new phase in bipolar affective disorder. Bipolar Disorders, 5(5), 356-361.

[7] Griffin, D. W., \& Bartholomew, K. (1994). The metaphysics of measurement: The case of adult attachment. In: K. Bartholomew, \& D. Perlman (Eds.) Advances in Personality Relationship vol. 5 Attachment processes in adulthood. London: Jessica Kingsley Publishers, pp17-52.

[8] Grouzet, F., Kasser, T., Ahuvia, A., Dols, J. M. F., Kim, Y., Lau, S., Ryan, R. M., Saunders, S., Schmuck, P., \& Sheldon, K. M. (2005). The structure of goal contents across 15 cultures. Journal of Personality and Social Psychology, 89, 800-816.

[9] Haller, M., \&Hadler, M. (2006). How social relations and structures can produce happiness and unhappiness: An international comparative analysis. Social Indicators Research, $75,169-216$.

[10] Hammen, C. L., Burge, D., Daley, S. E., Davila, J., Paley, B., \& Rudolph, K. D. (1995). Interpersonal attachment cognitions and prediction of symptomatic responses to interpersonal stress. Journal of Abnormal Psychology, 104, 436-443.

[11] Kasser, T., \& Ryan, R. M. (1993). A dark side of the American dream: Correlates of financial success as a central life aspiration. Journal of Personality and Social Psychology, 65, 410-422.

[12] Kasser, T., \& Ryan, M. R. (1996). Further examining the American dream: Differential correlates of intrinsic and extrinsic goals. Personality and Social Psychology Bulletin, 22, 280-287.

[13] Kenny, M. E., Lomax, R., Brabeck, M., \& Fife, J. (1998). Longitudinal pathways linking adolescent report of maternal and paternal attachment to psychological wellbeing. Journal of Early Adolescence, 18, 221-243.

[14] Kim, Y., Kasser, T., \& Lee, H. (2003). Self-concept, aspirations, and well-being in South Korea and the United States. Journal of Social Psychology, 143, 277-290.

[15] Kitamura, T. (1993). Hospital Anxiety and Depression Scale. Archives of Psychiatric Diagnostics and Clinical Evaluation, 4, 371-372 (in Japanese).

[16] Liu, Q., Nagata, T., Shono, M., \& Kitamura, T. (2009). The effects of adult attachment style and negative life events on daily depression: A sample of Japanese university students. Journal of Clinical Psychology, 65, 639-652.

[17] Markus, H. R., \&Kitayama, S. (1991). Culture and the self: Implications for cognition, emotion, and motivation. Psychological Review, 98, 224-253.

[18] Markus, H., \&Wurf, E. (1987). The dynamic self-concept: A social psychological perspective. Annual Review of Psychology, 38, 299-337. 
[19] Massey, E. K., Gebhardt, W. A., \&Garnefski, N. (2008). Adolescent goal content and pursuit: A review of the literature from the past 16 years. Developmental Review, 28, 421-460.

[20] Matsudaira, T., Igarashi, H., Kikuchi, H., Kano, R., Mitoma, H., Ohuchi, K., \& Kitamura, T. (2009). Factor structure of the Hospital Anxiety and Depression Scale in Japanese psychiatric outpatient and student populations. Health and Quality of Life Outcomes, 7, 42 .

[21] Mykletun, A., Stordal, E., \& Dahl, A. A. (2001). Hospital Anxiety and Depression (HAD) scale: Factor structure, item analyses and internal consistency in a large population. British Journal of Psychiatry, 179, 540-544.

[22] Oishi, S., Diener, E. F., Lucas, R. E., \&Suh, E. M. (1999). Cross-cultural variations in predictors of life satisfaction: Perspectives from needs and values. Personality and Social Psychology Bulletin, 25, 980-990.

[23] Oishi, S., Schimmack, U., Diener, E., Suh, E. M. (1998). The measurement of values and individualism-collectivism. Personality and Social Psychology Bulletin, 24, 1177-1189.

[24] Papini, D. R., \&Roggman, L. A. (1992). Adolescent perceived attachment to parents in relation to competence, depression and anxiety: A longitudinal study. Journal of Early Adolescence, 12, 420-440.
[25] Petersen, A. C., Sarigiani, P. A., \& Kennedy, R. E. (1994). Adolescent depression: Why more girls? Journal of Youth and Adolescence, 20, 247-269.

[26] Plagnol, A. C., \&Easterlin, R. A. (2008). Aspirations, attainments, and satisfaction: Life cycle differences between American women and men. Journal of Happiness Studies, 9, 601-619.

[27] Schermelleh-Engel1, K., Moosbrugger, H., \& Müller, H. (2003). Evaluating the fit of structural equation models: tests of significance and descriptive goodness-of-fit measures. Methods of Psychological Research Online, 8, 23-74.

[28] Schmuck, P., Kasser, T., \& Ryan, R. M. (2000). Intrinsic and extrinsic goals: Their structure and relationship to well-being in German and U.S. college students. Social Indicator Research, 50,225-241.

[29] Welzel, C., \&Inglehart, R. (2010). Agency, values, and well-being: A human development model. Social Indicators Research, 97,43-63.

[30] Zigmond, A. S., \&Snaith, R. P. (1983). The Hospital Anxiety and Depression scale. ActaPsychiatricaScandinavica, 67, 361-370. 